Research Article

\title{
Pan-Cancer Analysis Reveals FH as a Potential Prognostic and Immunological Biomarker in Lung Adenocarcinoma
}

\author{
Heng Zhang $\mathbb{D}^{1}$ Qiang Ju $\mathbb{D},^{2}$ Jing Ji $\mathbb{D}^{1},{ }^{1}$ and Yanjie Zhao $\mathbb{D}^{1}$ \\ ${ }^{1}$ School of Public Health, Qingdao University, Qingdao, Shandong, China \\ ${ }^{2}$ Department of Blood Transfusion, The Affiliated Hospital of Qingdao University, Qingdao, Shandong, China
}

Correspondence should be addressed to Yanjie Zhao; zhaoyj@qdu.edu.cn

Received 7 September 2021; Accepted 11 October 2021; Published 26 October 2021

Academic Editor: Ming Li

Copyright (C) 2021 Heng Zhang et al. This is an open access article distributed under the Creative Commons Attribution License, which permits unrestricted use, distribution, and reproduction in any medium, provided the original work is properly cited.

Fumarate hydratase $(\mathrm{FH})$ is an important enzymatic component in the tricarboxylic acid cycle. Studies have reported that $\mathrm{FH}$ plays an important role in hereditary leiomyomatosis and renal cell cancer (HLRCC). However, the role of FH in human different cancers remains unknown. This study is aimed at analyzing the prognostic value of FH and demonstrating the correlation between FH expression and tumor immunity. Results showed that FH was mutated or copy number varied in 27 types of cancer. FH mRNA was abnormally upregulated across various cancers. Survival analysis suggested high expression of FH was associated with poor prognosis in many cancer types, including lung adenocarcinoma (LUAD). Additionally, FH expression was associated with immune infiltration, including $\mathrm{B}$ cells, $\mathrm{CD} 4^{+} \mathrm{T}$ cells, $\mathrm{CD} 8^{+} \mathrm{T}$ cells, neutrophils, macrophages, and dendritic cells, especially in liver hepatocellular carcinoma (LIHC), LUAD, and lung squamous cell carcinoma (LUSC). Moreover, FH expression showed a strong correlation with immune checkpoint markers in LUAD and testicular germ cell tumors (TGCT). These results indicate that FH is an immunotherapeutic target and a potential prognostic biomarker in LUAD.

\section{Introduction}

Cancer is the leading cause of death worldwide, and most existing therapies are low effective [1-3]. Pan-cancer analyses can help us to find common and different characteristics of human malignant tumors [4] and provide novel ideas for the clinical treatment of tumors [5], for example, applying pan-cancer analysis to reveal that immune infiltration influences radiotherapy outcomes [6] and to explore the association between matrisome genes and tumors [7]. In addition, pan-cancer analysis can be used to find valuable prognostic biomarkers [8-10]. Therefore, pan-cancer analysis is an important method for identifying new diagnostic biomarkers and developing more effective molecular targets for cancer treatment.

Fumarate hydratase $(\mathrm{FH})$ is an enzymatic component of the tricarboxylic acid cycle catalyzing fumarate to malate [11]. A growing number of studies have shown that $\mathrm{FH}$ is involved in the occurrence and development of certain cancers. For instance, patients with $\mathrm{FH}$ gene mutations have a very high risk of hereditary leiomyomatosis and HLRCC
[12]. And gastric cancer patients with high $\mathrm{FH}$ expression had a higher risk of death than those with low FH expression [13]. In addition, the loss of $\mathrm{FH}$ and the accumulation of fumarate elicit an epithelial-to-mesenchymal-transition (EMT) to promote cancer metastasis $[14,15]$. However, the role of FH in pan-cancers needs further study.

The occurrence and development of cancer are closely related to the surrounding stroma. Immune cells play important roles in the occurrence and progression of tumors and are crucial parts of tumor stroma $[16,17]$. Tumorassociated macrophages (TAMs) are important immune cells in the tumor microenvironment and play protumoral or antitumoral roles $[18,19]$. Therefore, the study of tumor immune microenvironment can provide new clues for understanding the mechanism of tumor occurrence and development and has important value for the clinical treatment of tumors. However, the current research on the role of FH in tumor immunity is still limited.

In this study, we analyzed the expression of $\mathrm{FH}$ and evaluated its prognostic value in 33 cancer types. More importantly, we explored the relationship between FH expression 
and various tumor immunities. Our results provide new insights into the role of $\mathrm{FH}$ in tumors, suggesting that $\mathrm{FH}$ is related to the immune infiltration of a variety of tumors and is a potential prognostic biomarker, especially in lung adenocarcinoma (LUAD).

\section{Materials and Methods}

2.1. Pan-Cancer Analysis of Mutational Data of FH. The mutation and amplification levels of $\mathrm{FH}$ in human cancers were evaluated by cBioPortal database (http://www .cbioportal.org/, v3.6.20). By using TCGA database (https:// gdc.cancer.gov/access-data/gdc-data-transfer-tool, v23.0), we obtained the mutation levels of five mismatch repair (MMR) genes (MLH1, MSH2, MSH6, PMS2, and EPCAM). The correlation between FH level and MMR gene mutation level was explored by the Pearson correlation analysis.

2.2. Patient Datasets and FH Expression Analysis. The data of the FH expression in tumor and normal tissues of 33 types of cancers were obtained from the Genotype Tissue Expression (GTEx) (https://gtexport.org/home/, v8) and The Cancer Genome Atlas (TCGA). Clinical annotations and RNA sequencing data of 33 cancer types (ACC: adrenocortical carcinoma; BLCA: bladder urothelial carcinoma; BRCA: breast invasive carcinoma; CESC: cervical squamous cell carcinoma; CHOL: cholangiocarcinoma; COAD: colon adenocarcinoma; DLBC: lymphoid neoplasm diffuse large B cell lymphoma; ESCA: esophageal carcinoma; GBM: glioblastoma multiforme; LGG: brain lower grade glioma; HNSC: head and neck squamous cell carcinoma; KICH: kidney chromophobe; KIRC: kidney renal clear cell carcinoma; KIRP: kidney renal papillary cell carcinoma; LAML: acute myeloid leukemia; LIHC: liver hepatocellular carcinoma; LUAD: lung adenocarcinoma; LUSC: lung squamous cell carcinoma; MESO: mesothelioma; OV: ovarian serous cystadenocarcinoma; PAAD: pancreatic adenocarcinoma; PCPG: pheochromocytoma and paraganglioma; PRAD: prostate adenocarcinoma; READ: rectum adenocarcinoma; SARC: sarcoma; SKCM: skin cutaneous melanoma; STAD: stomach adenocarcinoma; TGCT: testicular germ cell tumors; THCA: thyroid carcinoma; THYM: thymoma; UCEC: uterine corpus endometrial carcinoma; UCS: uterine carcinosarcoma; and UVM: uveal melanoma) were obtained from TCGA. All data were normalized as previously described $[20,21]$.

2.3. Cell Culture and Reagents. BEAS-2B, 16HBE, A549, and $\mathrm{H} 460$ were obtained from the American Type Culture Collection (ATCC, Manassas, USA). 16HBE, BEAS-2B, and H460 were cultured in MEM medium (HyClone, Utah, USA) with 10\% fetal bovine serum (FBS, Gibco, Amarillo, TX). A549 was cultured in RPMI 1640 medium (Cytiva, Utah, USA) with $10 \%$ fetal bovine serum. All cells were cultured in $37^{\circ} \mathrm{C}$ humidified incubator with $5 \% \mathrm{CO}_{2}$.

2.4. RNA Isolation and Real-Time PCR Analysis. According to the manufacturer's protocol, total RNA was isolated from cell lines by using TRIzol reagent (Invitrogen, USA). Complementary DNA (cDNA) was obtained using a PrimeScript RT reagent kit (TaKaRa, Japan). Real-time PCR was per- formed using TB Green Premix Ex Taq II (TaKaRa, Japan) in a Light Cycler 480 II Real-Time PCR system. Glyceraldehyde phosphate dehydrogenase (GAPDH) was employed as a control for normalization. The primers were shown as follows: $\mathrm{FH}$ forward $5^{\prime}$-CCGCTGAAGTAAACCAGGATT ATG- $3^{\prime}$ and $\mathrm{FH}$ reverse $5^{\prime}$-ATCCAGTCTGCCATACCAC GAG-3'; and GAPDH forward $5^{\prime}$-GTCTCCTCTGACTT CAACAGCG- $3^{\prime}$ and GAPDH reverse $5^{\prime}$-ACCACCCTGTT GCTGTAGCCAA-3.

2.5. Correlation between FH Expression Level and Patients' Prognosis. The relationship between $\mathrm{FH}$ expression and OS in 33 types of cancer was analyzed by forest plots and the Kaplan-Meier curves. The hazard ratio (HR) and log-rank $P$ values were acquired by univariate survival analysis.

2.6. Association between FH and Tumor Immunity. The Tumor Immune Estimation Resource (TIMER) database (https://cistrome.shinyapps.io/timer/, v2.0) was used to obtain immune infiltrating cell scores for 33 cancer types. The associations between FH levels and 6 immune infiltrates cells-B cell, $\mathrm{CD}^{+} \mathrm{T}$ cell, $\mathrm{CD} 8^{+} \mathrm{T}$ cell, neutrophil cell, macrophage cell, and dendritic cell-were evaluated by the Spearman correlation analysis. Moreover, using the Pearson correlation analysis, we examined the correlation between FH level and immune checkpoint marker level.

2.7. Statistical Analysis. The expression level of FH in different tissues was analyzed by $t$ test. The univariate survival analysis and Kaplan-Meier survival analysis were used to analyze the correlation between $\mathrm{FH}$ expression and patients' overall survival. $P<0.05$ were considered significant for all statistical analysis.

\section{Results}

3.1. Genomic Alterations of FH in Human Pan-Cancer. As we all know, genomic mutation is closely related to tumorigenesis [22]. Using the cBioPortal database, we identified genomic alterations of $\mathrm{FH}$ in 32 cancers, including mutations and copy number variations. As a result, FH was mutated or copy number varied in 27 cancers. The results showed that $\mathrm{FH}$ mutation frequencies are high in UCEC, BLCA, HNSC, and LAML. Furthermore, FH amplification was one of the significant single factors for alteration in CHOL, USC, PCPG, ESCA, and KIRC (Figure 1(a)). In addition, $73 \mathrm{FH}$ mutations were identified across pan-cancer, and all of them (100\%) were missense (Figure 1(b)).

DNA mismatch repair (MMR) maintains genomic stability [23]. Mutations in MMR gene might cause defective mismatch repair, leading to genomic alterations of some genes [24]. Next, we investigated the correlation of four MMR genes' mutation and FH. As shown in Figure 1(c), in most types of cancers, such as LUAD, BLCA, and LUSC, FH expression was significantly related with the mutation level of MMR genes. We next explored the relationship between FH expression and tumor mutational burden (TMB) level. FH expression was associated with TMB in BRCA, COAD, HNSC, LGG, LIHC, LUAD, PAAD, PRAD, SKCM, STAD, THYM, 


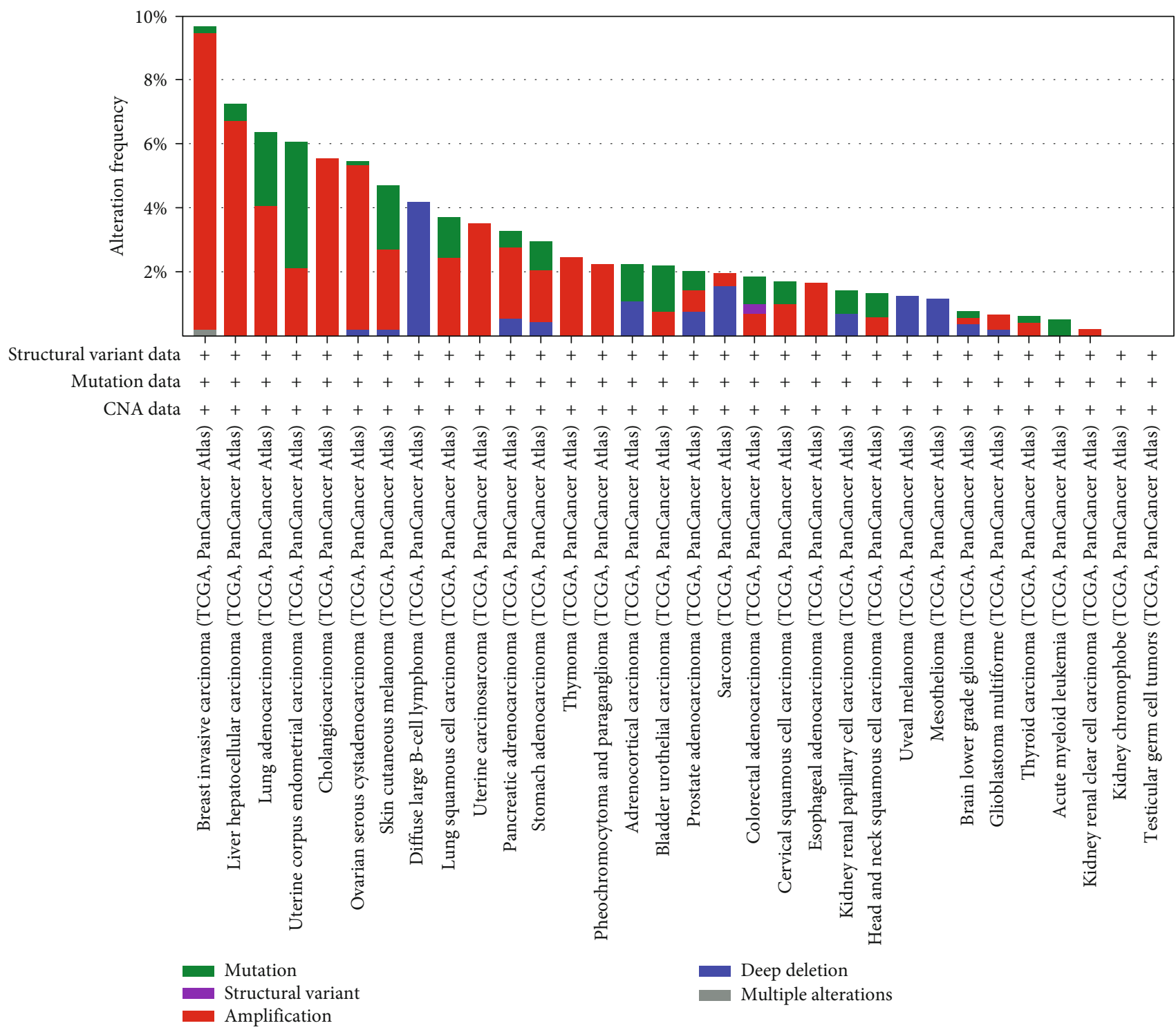

(a)
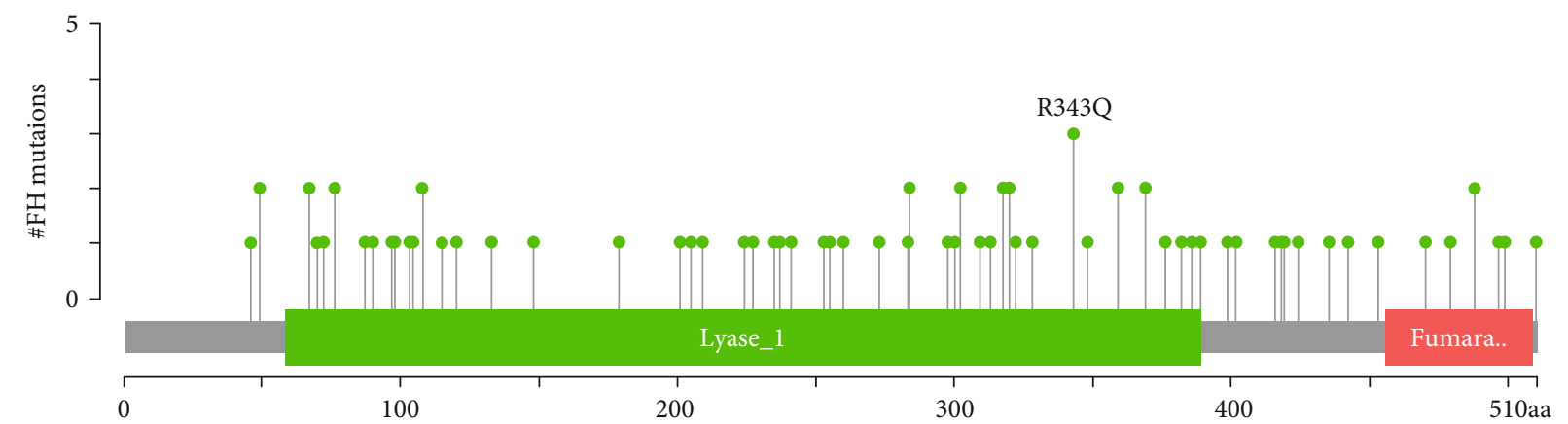

(b)

FIgUre 1: Continued. 


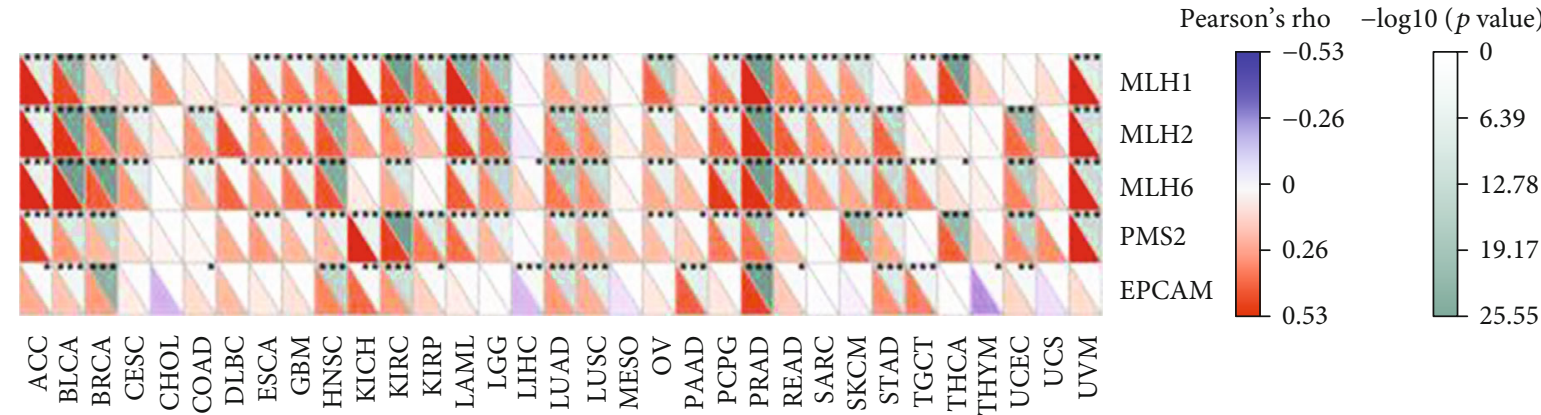

(c)

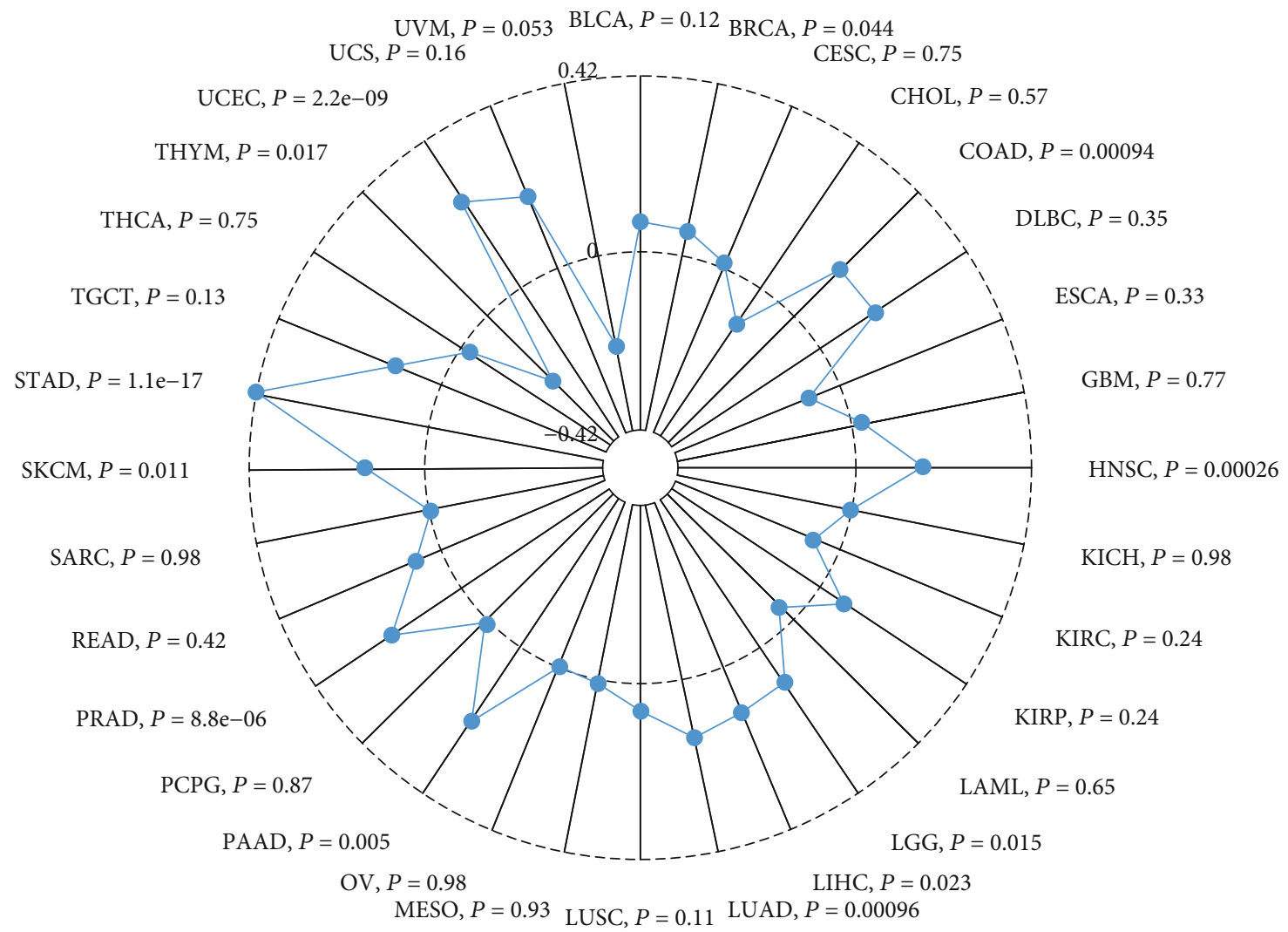

(d)

Figure 1: Genomic alterations of FH in human pan-cancer. (a) The alteration frequency of FH in human pan-cancer. (b) The types and distributions of FH mutations. $X$-axis: amino acid; $Y$-axis: numbers of FH mutations; green/red box: RNA recognition motif (190-248aa, 376-444aa, and 471-527aa); \#: number of FH mutations. (c) The association between FH expression level and four MMR genes mutation. (d) Radar map showing the correlation between FH expression and TMB. ${ }^{*} P<0.05,{ }^{* *} P<0.01,{ }^{* * *} P<0.001$.

and UCEC (Figure 1(d)). All these results indicate FH shows genomic alterations in many cancers.

3.2. The mRNA Expression of FH in Human Pan-Cancer. Next, the FH level between tumor tissues and normal tissues in 20 types of cancers was obtained from TCGA database. $\mathrm{FH}$ was overexpressed in BRCA, ESCA, GBM, LIHC, LUAD, LUSC, PRAD, STAD, and UCEC tissues compared with normal tissues (Figure 2(a)). In addition, we combined the GTEx database to expand the normal tissue data. Furthermore, the expression level of FH in 27 tumors was analyzed. As shown in Figure 2(b), FH was upregulated in 21 types of cancer tissues, including CC, BLCA, BRCA, CESC, COAD,
ESCA, GBM, KICH, LGG, LIHC, LUAD, LUSC, OV, PAAD, PRAD, SKCM, STAD, TGCT, THCA, UCEC, and UCS. These results suggest that $\mathrm{FH}$ is abnormally upregulated in various cancers.

3.3. Prognostic Value Analysis of FH in Human Pan-Cancer. Next, we investigated whether abnormal expression of $\mathrm{FH}$ affects patients' prognosis. By univariate survival analysis, we found that $\mathrm{FH}$ expression was associated with patients' OS in 8 cancer types, including ACC, KICH, KIRC, KIRP, LAML, LGG, LUAD, and SKCM (Figure 3(a)). The Kaplan-Meier curves showed that increased $\mathrm{FH}$ expression was correlated with poor prognosis in 6 cancer types including ACC 


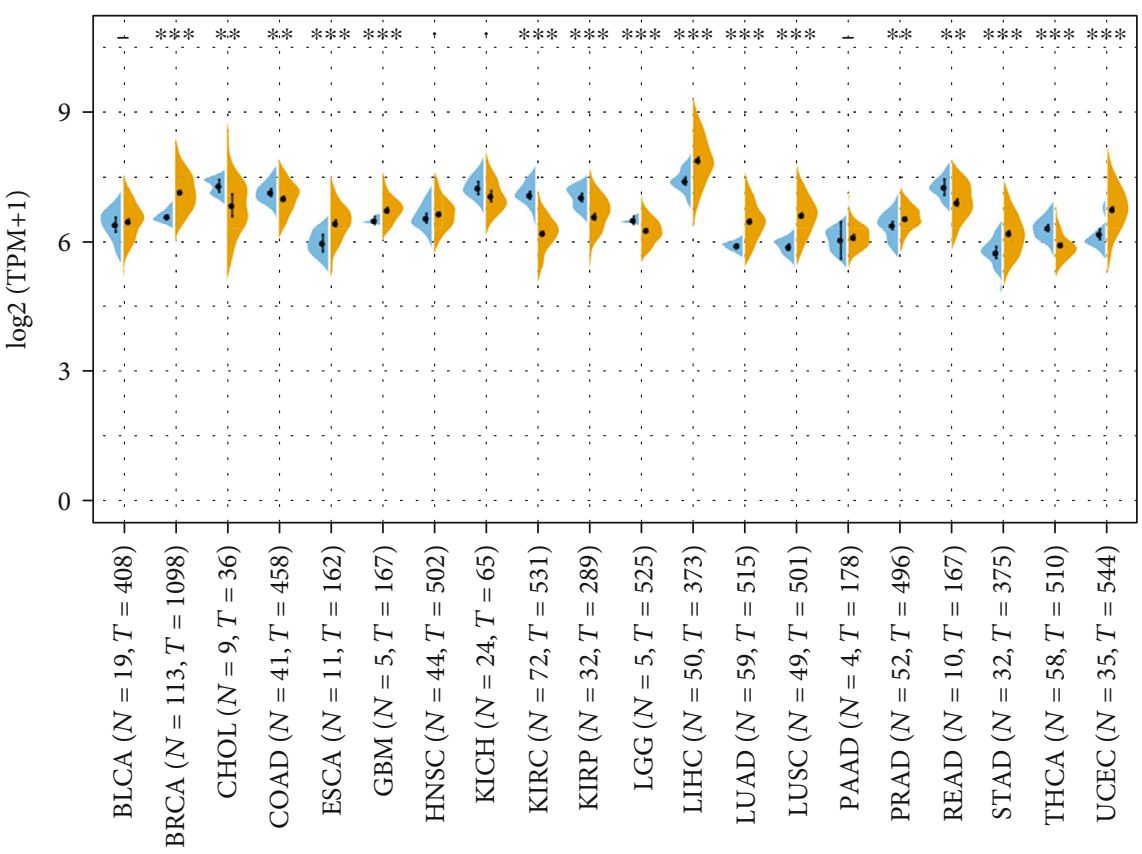

Group

- Normal

- Tumor

(a)

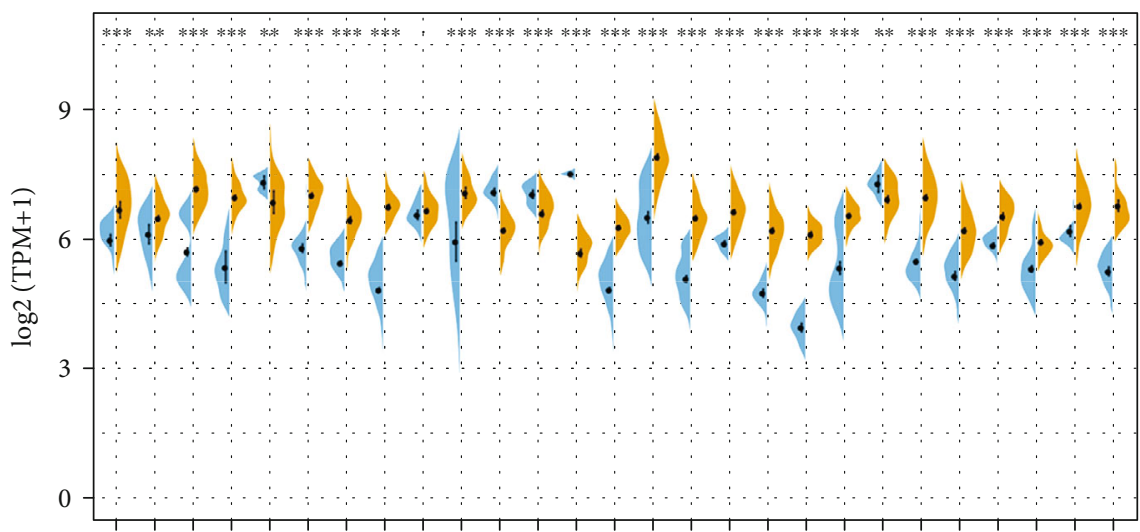

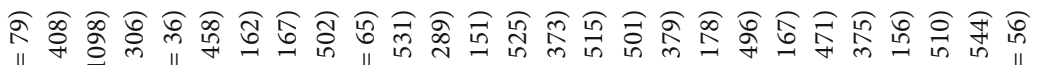

II

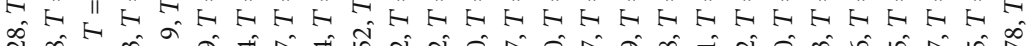

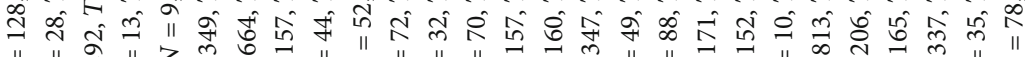

$\|$ iो $\|$ z

zる

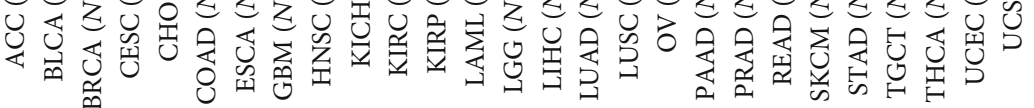

Group

- Normal

- Tumor

(b)

Figure 2: Expression level of FH in different cancer types. (a) Expression level of FH in tumor and normal tissues from TCGA database. (b) Expression level of FH in tumor and normal tissues from TCGA and the GTEx database. ${ }^{*} P<0.05,{ }^{* *} P<0.01,{ }^{* * *} P<0.001$.

$(P=0.00069, \mathrm{HR}=1.01)$, KICH $(P<0.0001, \mathrm{HR}=1.02)$, LAML $(P<0.0001, \mathrm{HR}=1.02)$, LGG $(P<0.0001, \mathrm{HR}=1)$, LUAD $(P=0.014, \mathrm{HR}=1)$, and SKCM $(P<0.0001, \mathrm{HR}=1)$.
However, KIRC $(P<0.0001, \mathrm{HR}=0.99)$ and $\mathrm{KIRP}(P=$ $0.00016, \mathrm{HR}=0.99)$ were exceptions where $\mathrm{FH}$ overexpression indicated a better prognosis (Figure 3(b)). 


$\begin{array}{lcc} & \text { HR } & P \text { value } \\ \text { ACC } & 1.01(1 \sim 1.01) & 0.02900 \\ \text { BLCA } & 1(1 \sim 1.01) & 0.15000 \\ \text { BRCA } & 1(1 \sim 1) & 0.25000 \\ \text { CESC } & 1(0.99 \sim 1) & 0.42000 \\ \text { CHOL } & 1(1 \sim 1.01) & 0.18000 \\ \text { COAD } & 1(0.99 \sim 1) & 0.11000 \\ \text { DLBC } & 1.01(0.99 \sim 1.03) & 0.23000 \\ \text { ESCA } & 1.01(1 \sim 1.01) & 0.16000 \\ \text { GBM } & 1(0.99 \sim 1.01) & 0.76000 \\ \text { HNSC } & 1(1 \sim 1.01) & 0.13000 \\ \text { KICH } & 1.02(1.01 \sim 1.03) & 0.00100 \\ \text { KIRC } & 0.99(0.99 \sim 1) & 0.00210 \\ \text { KIRP } & 0.99(0.98 \sim 1) & 0.00330 \\ \text { LAML } & 1.02(1.01 \sim 1.04) & 0.00016 \\ \text { LGG } & 1(1 \sim 1.01) & 0.00470 \\ \text { LIHC } & 1(1 \sim 1) & 0.47000 \\ \text { LUAD } & 1(1 \sim 1.01) & 0.01700 \\ \text { LUSC } & 1(1 \sim 1) & 0.38000 \\ \text { MESO } & 1(1 \sim 1.01) & 0.25000 \\ \text { OV } & 1(0.99 \sim 1) & 0.23000 \\ \text { PAAD } & 1(0.99 \sim 1.01) & 0.97000 \\ \text { PCPG } & 1(0.97 \sim 1.03) & 0.94000 \\ \text { PRAD } & 1(0.98 \sim 1.03) & 0.69000 \\ \text { READ } & 1(0.99 \sim 1.01) & 0.53000 \\ \text { SARC } & 1(1 \sim 1) & 0.90000 \\ \text { SKCM } & 1(1 \sim 1) & 0.00480 \\ \text { STAD } & 1(1 \sim 1.01) & 0.83000 \\ \text { TGCT } & 0.98(0.94 \sim 1.01) & 0.18000 \\ \text { THCA } & 1.01(0.99 \sim 1.03) & 0.24000 \\ \text { THYM } & 1.01(0.99 \sim 1.02) & 0.26000 \\ \text { UCEC } & 1(1 \sim 1) & 0.84000 \\ \text { UCS } & 1(0.99 \sim 1.01) & 0.90000 \\ \text { UVM } & 1.01(0.99 \sim 1.02) & 0.32000 \\ & (12 & \end{array}$

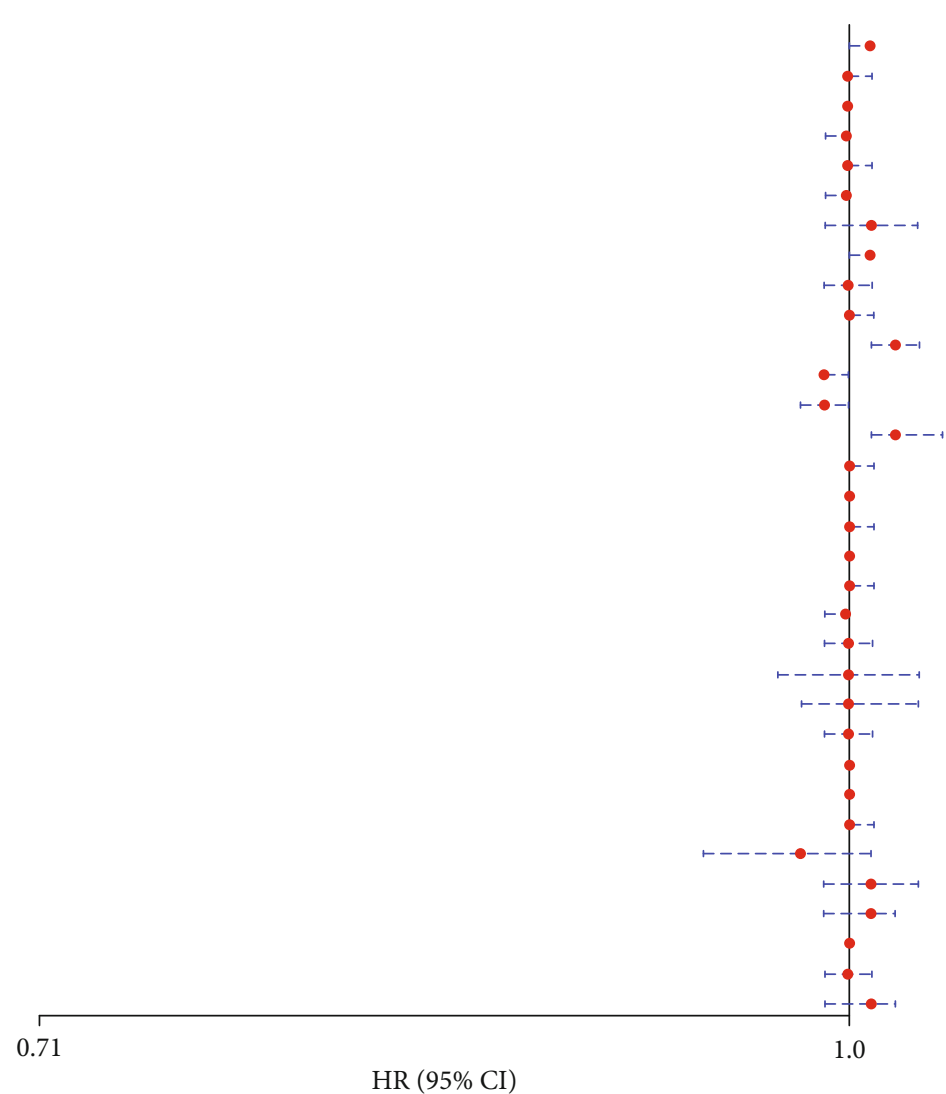

(a)
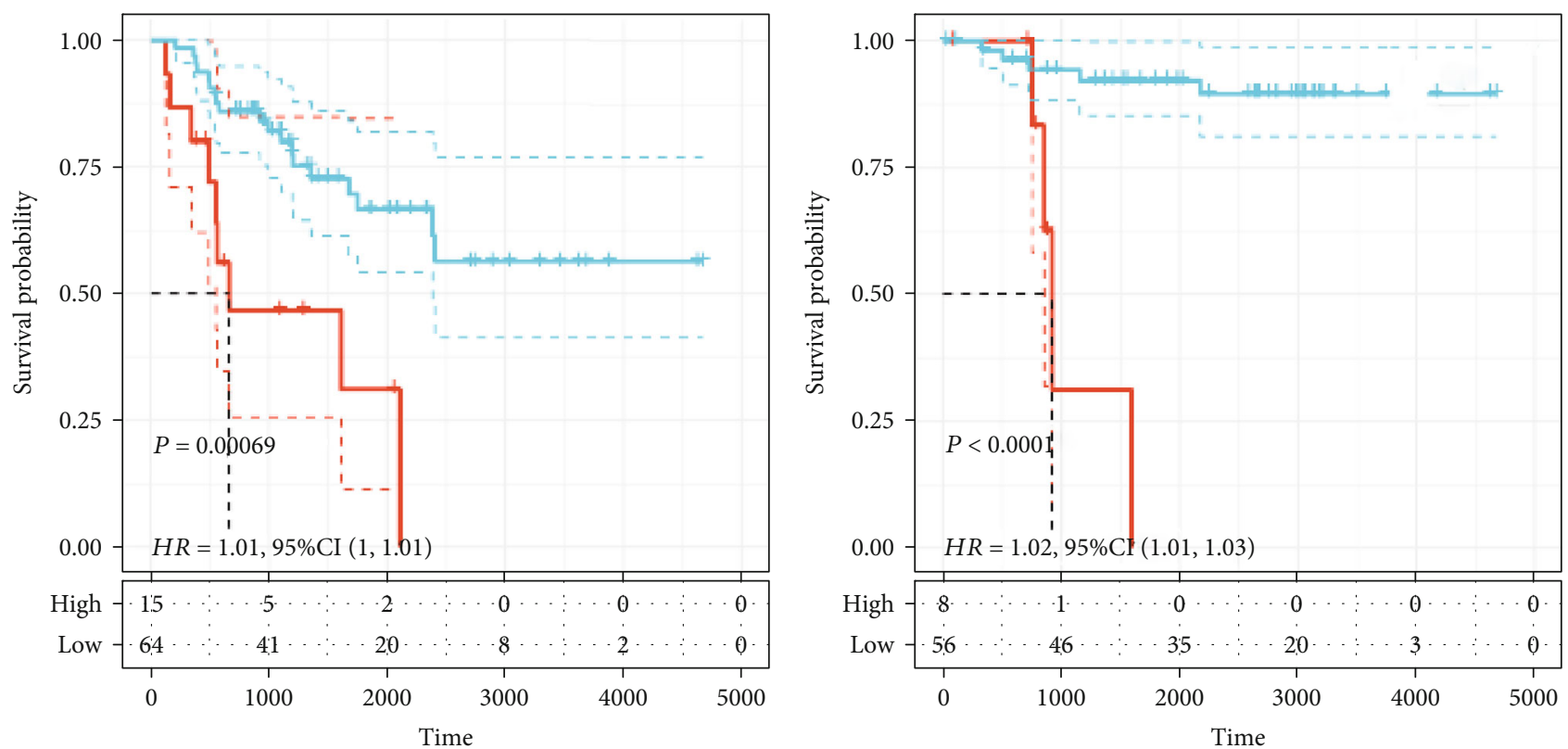

FH in ACC exp

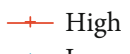

$\mathrm{FH}$ in $\mathrm{KICH} \exp$

+ High

+ Low

(b)

Figure 3: Continued. 

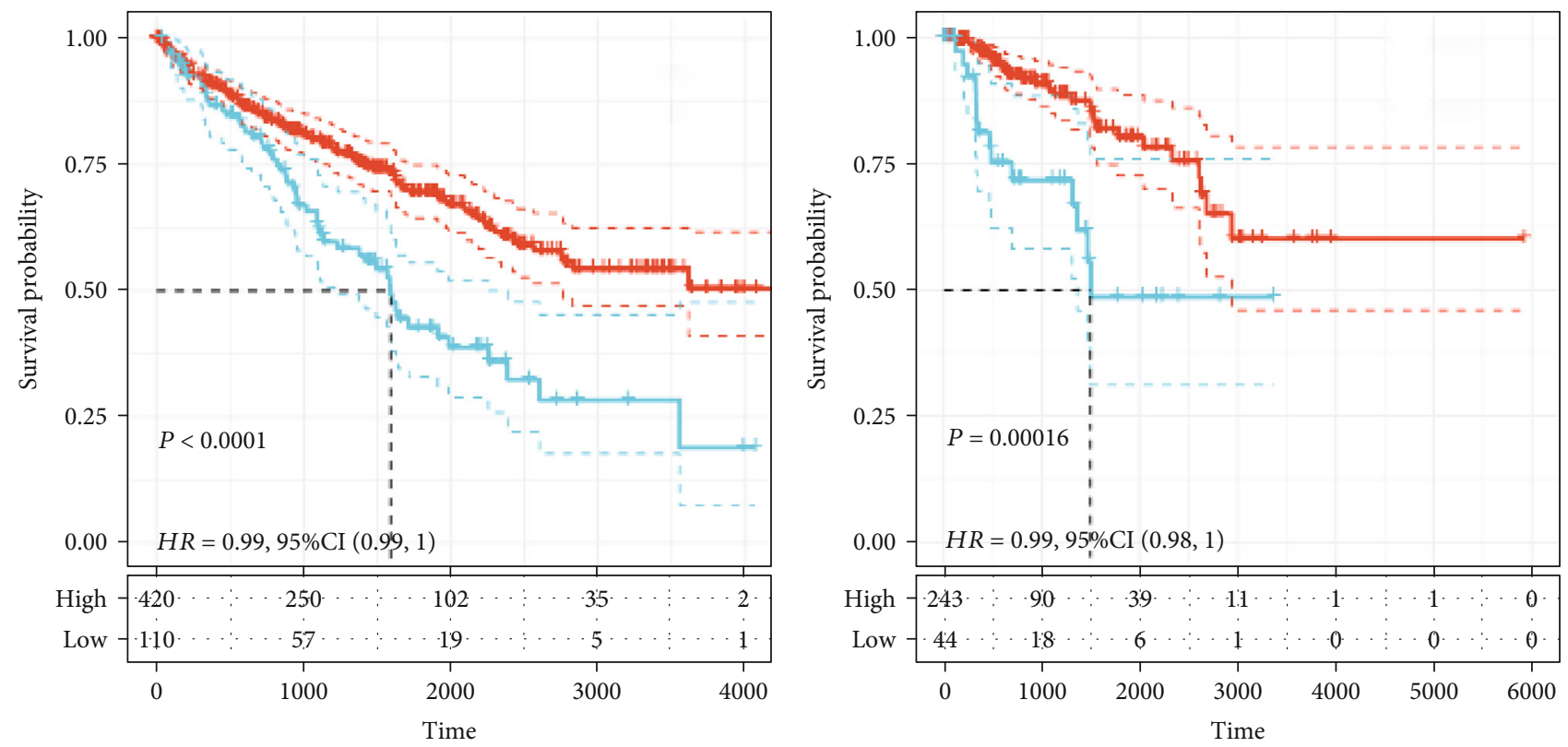

FH in KIRC exp

+ High

FH in KIRP exp

+ High

+ Low

(d)

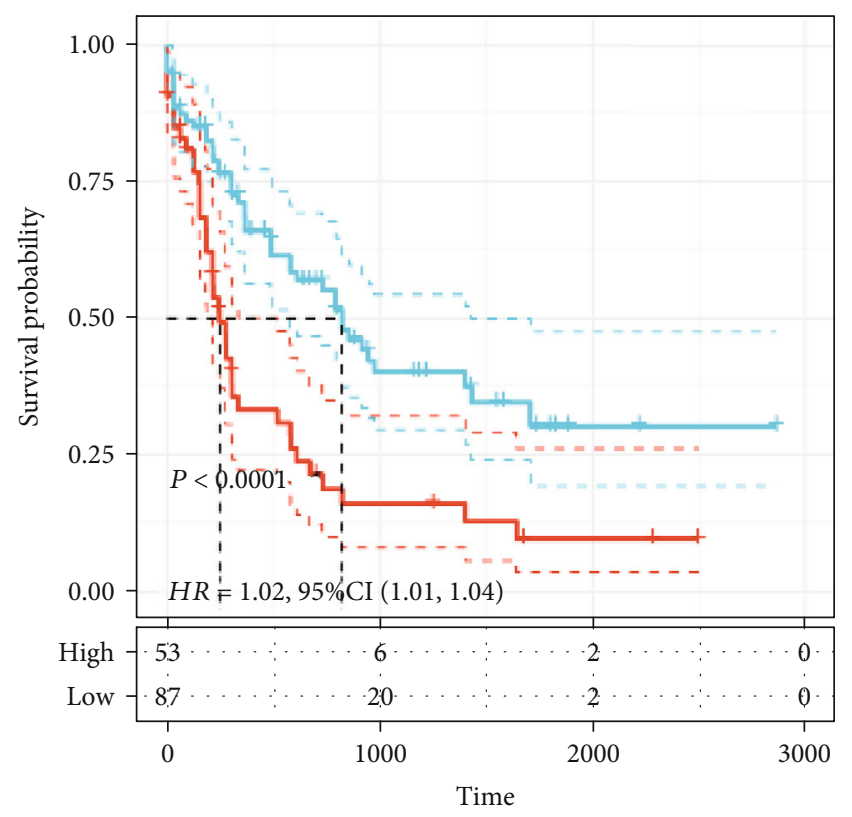

FH in LAML exp

+ High

+ Low

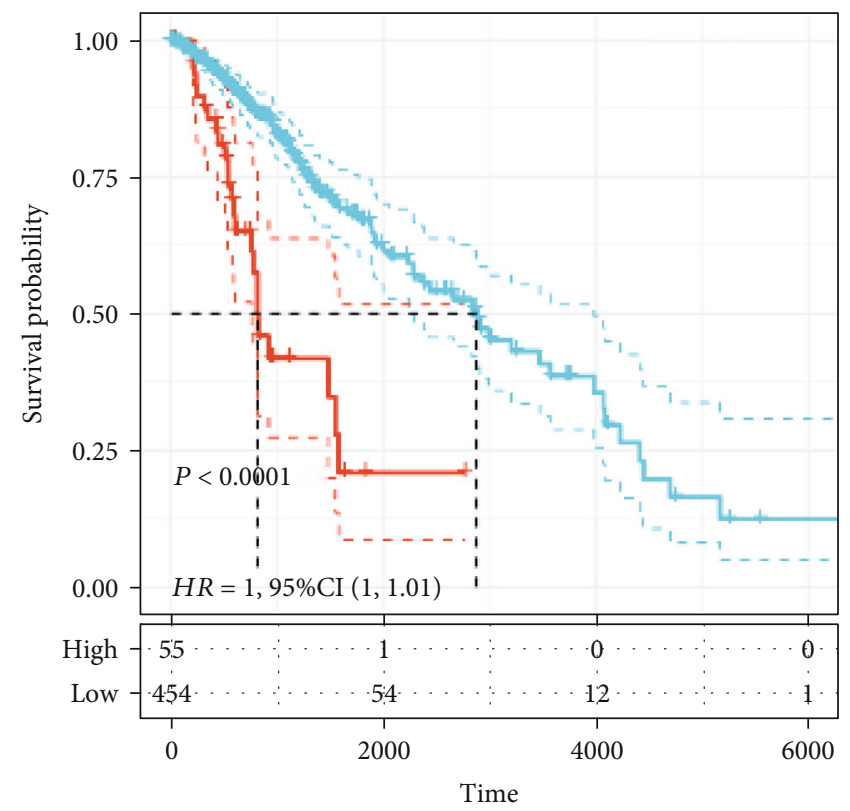

FH in LGG exp

+ High

+ Low

Figure 3: Continued. 


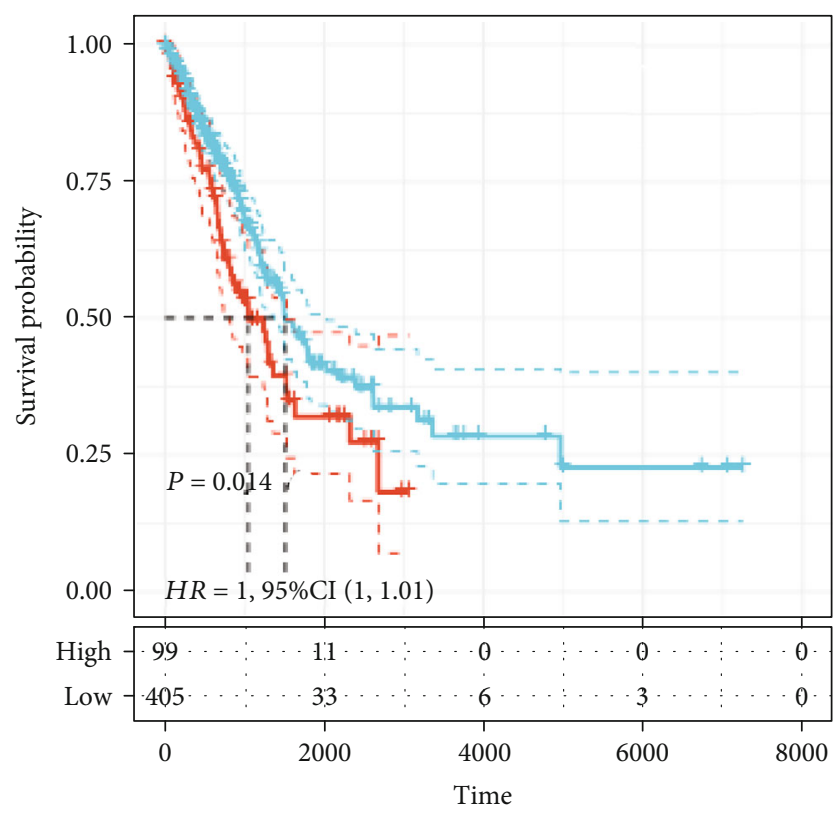

FH in LUAD exp

+ High

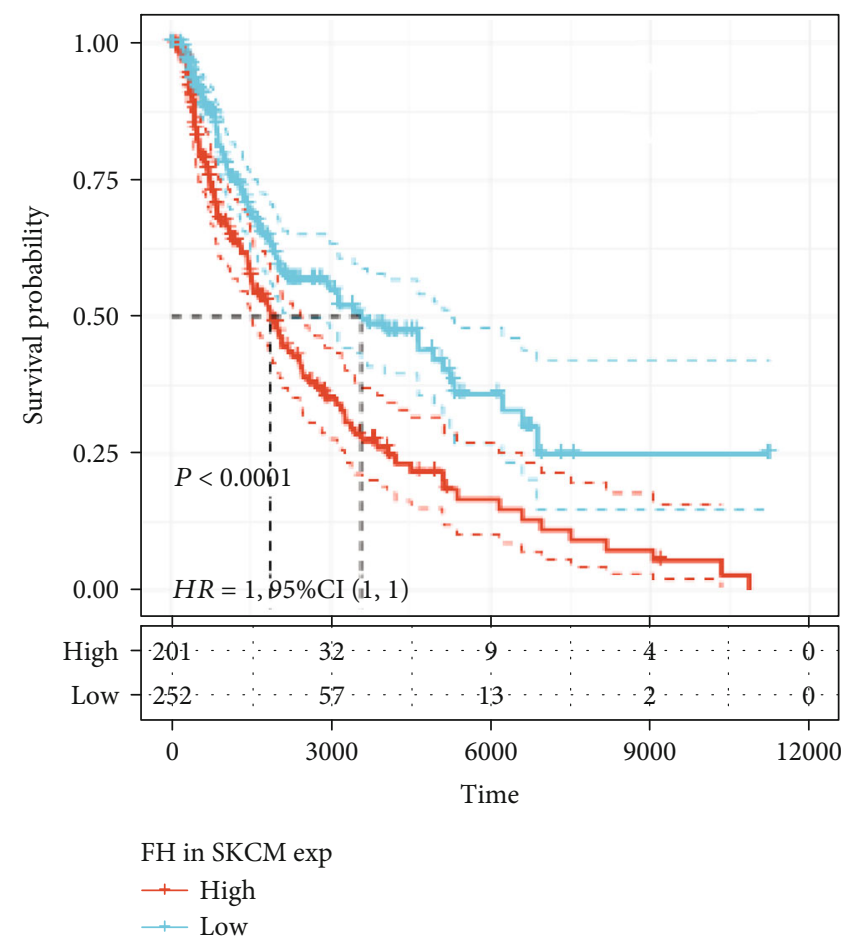

(i)

Figure 3: Association between FH expression level and patients' OS. (a) Forest plots of hazard ratios (HRs) of FH expression level in 33 tumor types. (b) The Kaplan-Meier analysis of the correlation between FH expression and patients' OS in ACC, KICH, KIRC, KIRP, LAML, LGG, LUAD, and SKCM.

3.4. The Association between FH Expression and Tumor Immunity. The immune cells in TME can affect patients' survival [25]. To explore the mechanism of $\mathrm{FH}$ affecting patients' prognosis, the correlation between $\mathrm{FH}$ expression and immune infiltration in pan-cancer was further investigated. First, we analyzed the scores of 6 types of immune cells (B cell, $\mathrm{CD}^{+} \mathrm{T}$ cell, $\mathrm{CD} 8^{+} \mathrm{T}$ cell, neutrophil cell, macrophage cell, and dendritic cell) from 33 cancer types through the TIMER database. Notably, FH level was significantly associated with 6 types of immune cells in LIHC, LUAD, and LUSC (Figure 4(a)). To quantify the immune and matrix components in cancers, the immune score (i.e., ImmuneScore), matrix score (i.e., StromalScore), and estimate score (i.e., ESTIMATEScore) were obtained. FH level was significant negatively associated with the ImmuneScore in SARC, BRCA, THCA, StromalScore in THCA, LUAD, TGCT, and ESTIMATEScore in THCA, LUAD, and SKCM (Figure 4(b)).

Next, we explored how FH affected immune cells infiltration. The correlation between $\mathrm{FH}$ expression and immune checkpoint gene expression was investigated. As shown in Figure 5, we found that in some cancers, especially in LUAD and TGCT, FH expression was significantly correlated with multiple immune checkpoint markers, such as BTLA, TNFRSF14, LAIR1, CD48, and CD28.

\section{Discussion}

Pan-cancer analysis can reveal similarities and differences in tumors. In recent years, many studies have used pan-cancer analysis to find biomarkers related to cancer prognosis and immunity $[26,27]$. FH protein participates in the tricarboxylic acid (TCA) cycle, where it catalyzes the reversible hydration of fumarate to malate [28]. At present, many studies have shown that TCA is closely related to the occurrence and development of cancer $[29,30]$. Therefore, the role of FH in cancer is worth exploring. Non-small cell lung cancer, especially lung adenocarcinoma, is a serious threat to human health and life. It is becoming more and more important to find new treatment methods and targets to improve the prognosis of lung cancer $[31,32]$. In this study, we explored the roles of FH in pan-cancer. On the one hand, we investigated genomic alterations of $\mathrm{FH}$ in pan-cancer and identified that there were mutations or copy number variations in $\mathrm{FH}$ genome. On the other hand, we found FH was upregulated in 21 types of cancers and related to patients' poor prognosis and immunity in LUAD. These results provide new clues for further research on the roles of $\mathrm{FH}$ in cancer.

Genomic instability, including genomic mutations and copy number variants, is the major cause of cancer development [33-36]. And research shows MMR gene mutations are closely related to tumorigenesis [37]. Our results showed FH genome mutation or copy number variation in many types of cancers. And FH expression was found significantly related with the mutation level of MMR genes and TMB level. In brief, our results showed that aberrant FH expression might play an important role in tumorigenesis.

FH has been reported to alter cancer cell migratory potential, and hopefully as a therapeutic target in renal cancer [38]. 

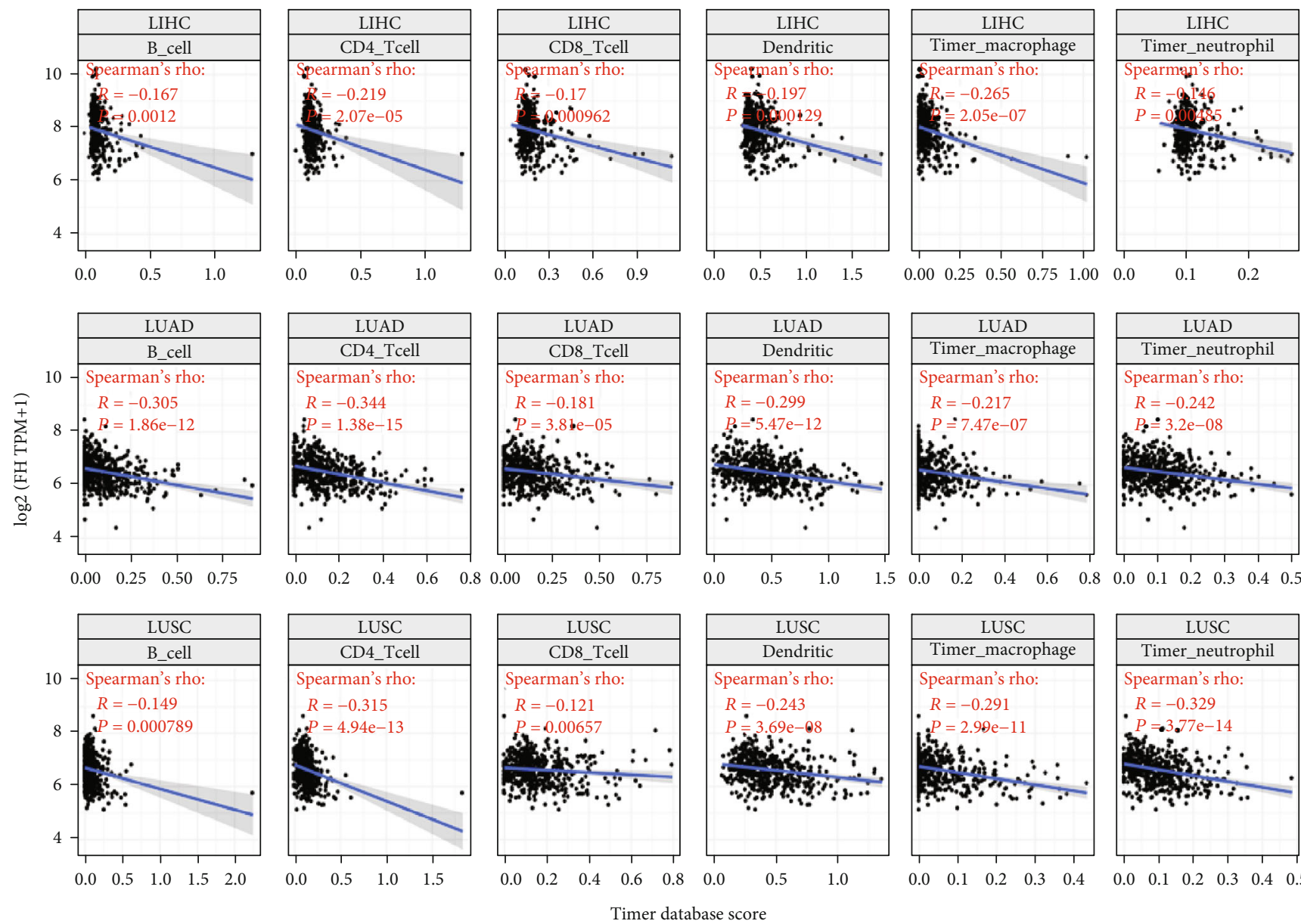

Timer database score

(a)

FIgURE 4: Continued. 

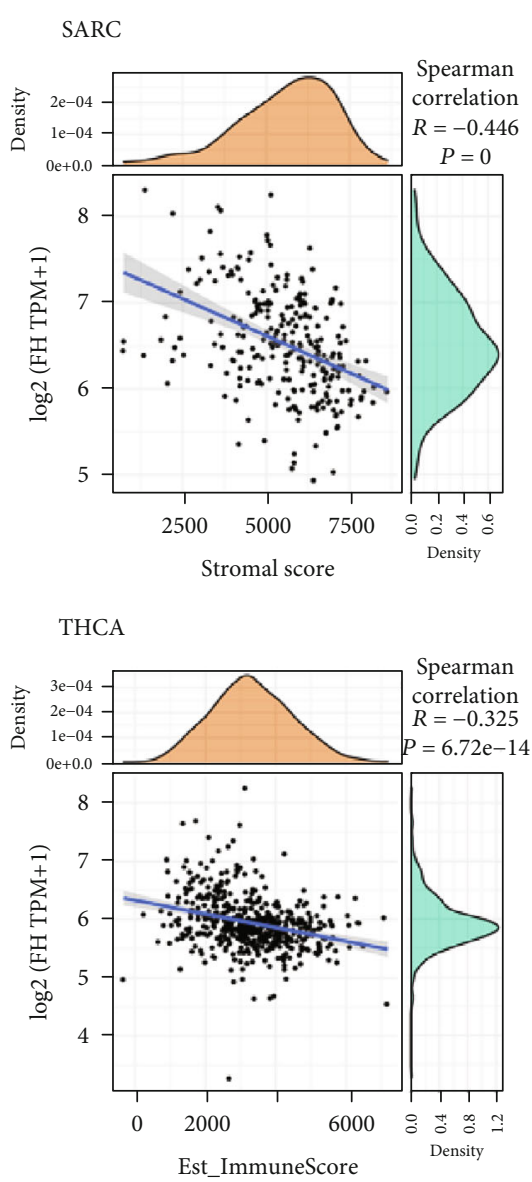

THCA

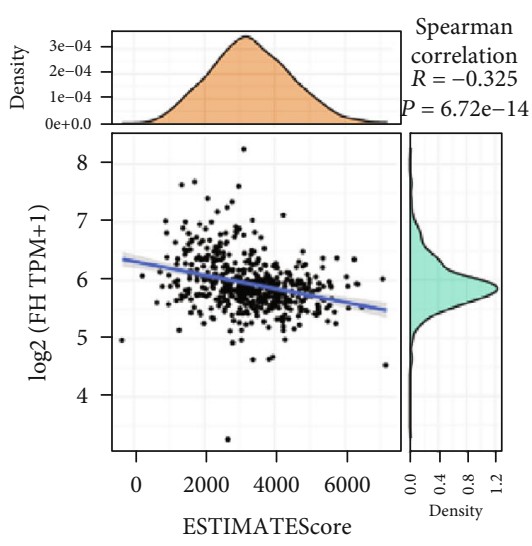

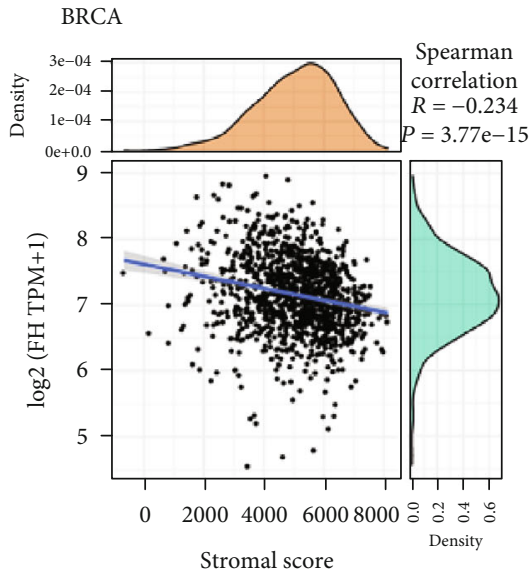
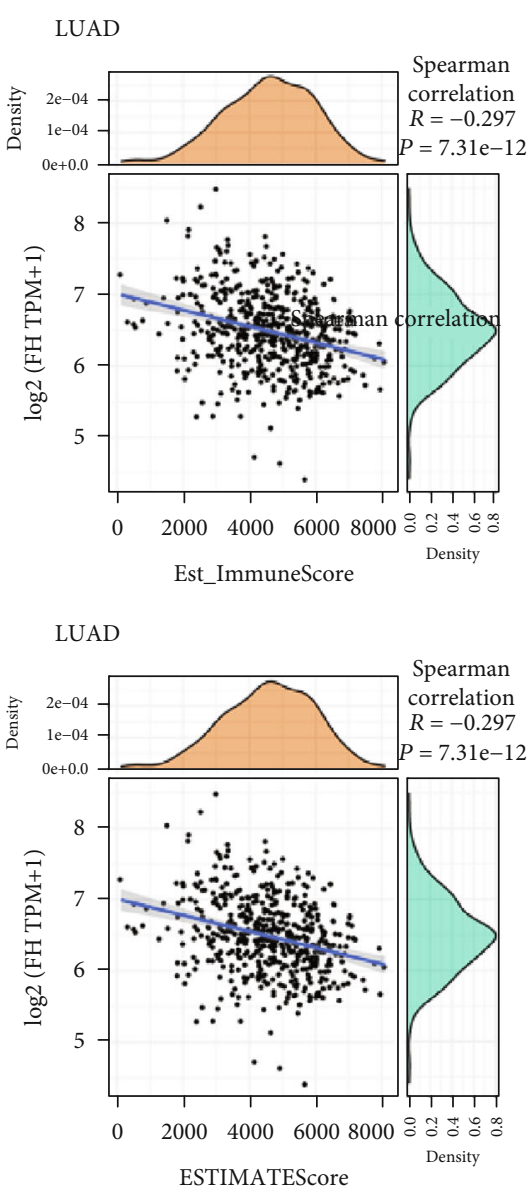

(b)
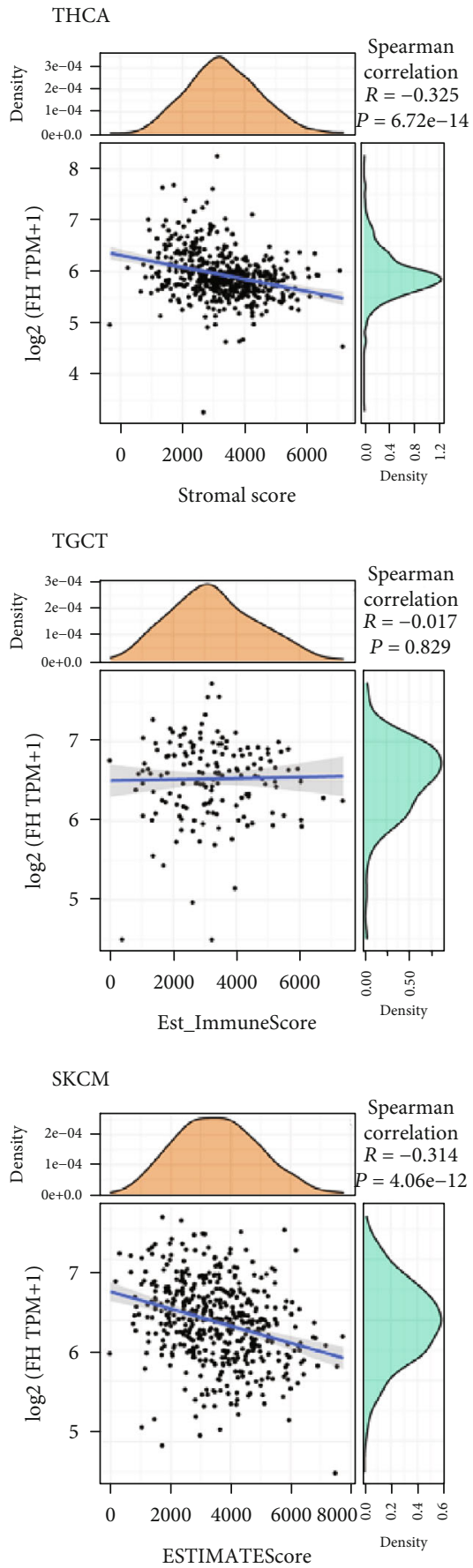

FIgURE 4: The association between FH expression and tumor immunity. (a) Correlation of FH expression with immune infiltration level of 6 types of immune cells in LIHC, LUAD, and LUSC. (b) Correlation analysis between FH expression and ImmuneScore/Stromal Score/ESTIMATEScore in human pan-cancer.

In addition, the inhibition of FH can improve the efficacy of cisplatin-mediated chemotherapy in GC [11]. However, the role of $\mathrm{FH}$ in other malignancies remains to be determined. In the present study, we found that $\mathrm{FH}$ was upregulated in 21 types of cancers tissues than in normal tissues. To further understand the roles of $\mathrm{FH}$ in cancer, we explored the prognostic value of FH in pan-cancer. A high expression level of
$\mathrm{FH}$ was associated with poor prognosis in several types of cancers, particularly in LUAD. And our experimental results also showed that FH level was significantly upregulated in lung cancer cell lines, including lung adenocarcinoma cell line A549 (Supplementary Figure 1). These results strongly indicated that $\mathrm{FH}$ is a significant gene in cancer and may be a potential prognostic marker in patients with LUAD. 


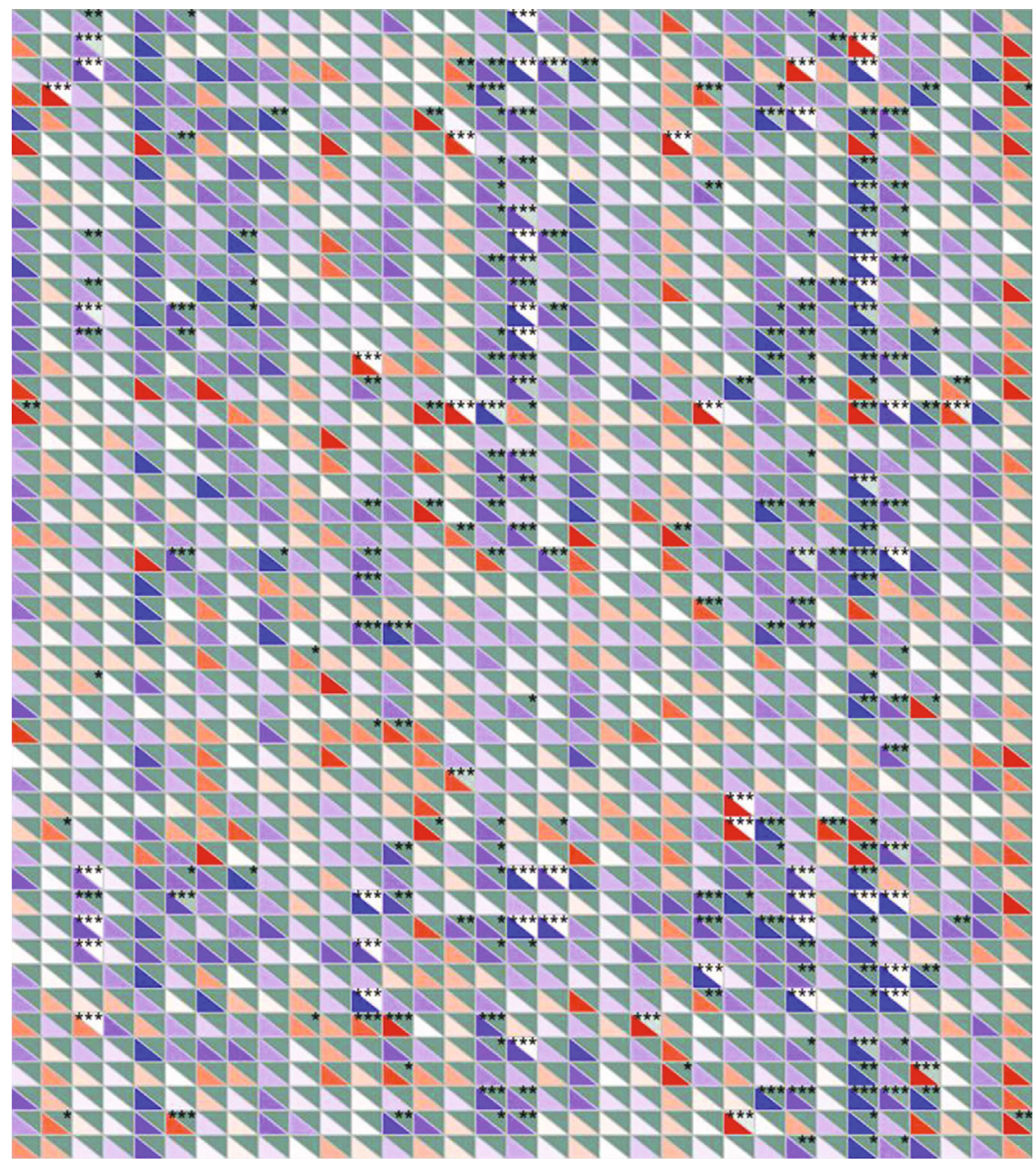

\begin{tabular}{l} 
BTLA \\
CD200 \\
TNFRSF14 \\
NRP1 \\
LAIR1 \\
TNFSF4 \\
CD244 \\
LAG3 \\
ICOS \\
CD40LG \\
CTLA4 \\
CD48 \\
CD28 \\
CD200R1 \\
HAVCR2 \\
ADORA2A \\
CD276 \\
KIR3DL1 \\
CD80 \\
PDCD1 \\
LGALS9 \\
CD160 \\
TNFSF14 \\
IDO2 \\
ICOSLG \\
TMIGD2 \\
VTCN1 \\
IDO1 \\
PDCD1LG2 \\
HHLA2 \\
TNFSF18 \\
BTNL2 \\
CD70 \\
TNFSF9 \\
TNFRSF8 \\
CD27 \\
TNFRSF25 \\
VSIR \\
TNFRSF4 \\
CD40 \\
TNFRSF18 \\
TNFSF15 \\
TIGIT \\
CD274 \\
CD86 \\
CD44 \\
TNFRSF9 \\
\\
\hline
\end{tabular}

Pearson's rho $\quad-\log 10$ ( $p$ value)

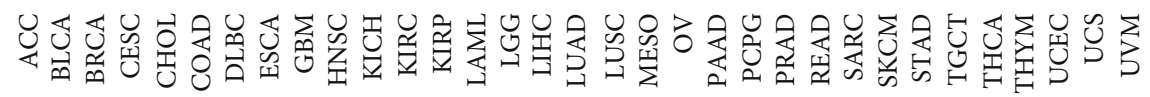

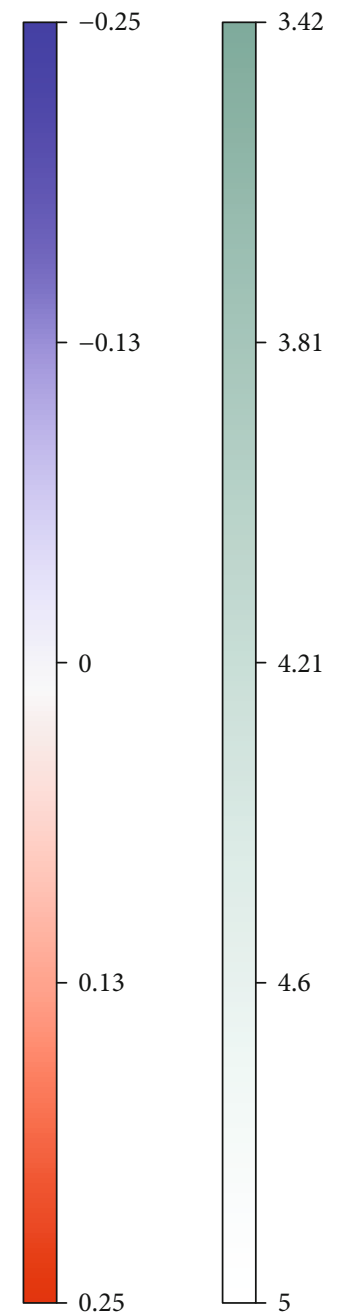

FIgURE 5: Correlation analysis of FH expression levels with 47 immune checkpoints in human pan-cancer. ${ }^{*} P<0.05,{ }^{* *} P<0.01,{ }^{* * *} P<0.001$.

Recently, tumor immune microenvironment has received extensive attention, and based on the characteristics of immune cells in the TME, immunotherapy was developed and applied to clinical treatment [39]. Tumor immune microenvironment is a double-edged sword: it can inhibit the development of tumors and can also provide favorable conditions for cancer cells to promote the development of tumors [40]. Tumor-infiltrating immune cells, such as B cells, T cells, neutrophils, macrophages, and dendritic cells, play a significant role in tumor immune microenvironment [41] and can affect the occurrence and development of tumors. For instance, B cells can secret immunoglobulins, promote T cell response, and kill cancer cells to inhibit tumor progression [42]. In advanced ovarian carcinoma, the presence of intratumoral $\mathrm{T}$ cells associates with improved survival of patients [43]. Neutrophils can stimulate $\mathrm{T}$ cell proliferation [44] to suppress tumor progression. Macrophages and dendritic cells are also closely related to tumor progression [45-47]. Interestingly, in our study, it was found that $\mathrm{FH}$ level was significantly negatively correlated with immune infiltrating cells in LUAD, LIHC, and LUSC. Moreover, we have noticed that immune checkpoint therapy is a hot spot in the treatment of cancer. For example, it can help us to define new means to treat pancreatic cancer [48] and has revolutionized lung cancer treatment paradigms [49]. So, we analyzed the correlations between FH level and immune checkpoint markers; the results showed that $\mathrm{FH}$ expression was significantly correlated with a variety of immune checkpoint markers in LUAD and TGCT. However, the results lack validation of clinical specimens, which is the limitation of the study. Overall, our results suggested $\mathrm{FH}$ is implicated in cancer immunity, particularly in LUAD.

\section{Conclusion}

In conclusion, we performed a pan-cancer analysis of the $\mathrm{FH}$ and elucidated the prognostic and immune significance of $\mathrm{FH}$ expression in human cancers. Our observations 
indicated FH may be an immunotherapeutic target and a potential prognostic biomarker, particularly in LUAD. This study provides new insights into the $\mathrm{FH}$ in pan-cancer and novel clues for further exploration of the mechanism of $\mathrm{FH}$ in cancer.

\section{Data Availability}

The datasets presented in this study can be found in online repositories. The names of the repositories can be found in the article material.

\section{Conflicts of Interest}

The authors disclose no conflicts.

\section{Acknowledgments}

This study was supported by the National Science Foundation for Young Scientists of China (grant no. 81802415 to Y.Z.). The authors sincerely thank all participants involved in this study.

\section{Supplementary Materials}

Supplementary Figure 1: Q-PCR analysis of FH expression in normal and lung adenocarcinoma cells. (Supplementary Materials)

\section{References}

[1] H. Sung, J. Ferlay, R. L. Siegel et al., "Global Cancer Statistics 2020: GLOBOCAN estimates of incidence and mortality worldwide for 36 cancers in 185 countries," CA: a Cancer Journal for Clinicians, vol. 71, no. 3, pp. 209-249, 2021.

[2] J. Wei, Y. Yan, X. Chen et al., "The roles of plant-derived triptolide on non-small cell lung cancer," Oncology Research, vol. 27, no. 7, pp. 849-858, 2019.

[3] Y. Yan, W. Su, S. Zeng et al., "Effect and mechanism of tanshinone I on the radiosensitivity of lung cancer cells," Molecular Pharmaceutics, vol. 15, no. 11, pp. 4843-4853, 2018.

[4] The Cancer Genome Atlas Research Network, J. N. Weinstein, E. A. Collisson et al., "The Cancer Genome Atlas pan-cancer analysis project," Nature Genetics, vol. 45, no. 10, pp. 11131120, 2013.

[5] J. N. Liu, X. S. Kong, T. Huang, R. Wang, W. Li, and Q. F. Chen, "Clinical implications of aberrant PD-1 and CTLA4 expression for cancer immunity and prognosis: a pan-cancer study," Frontiers in Immunology, vol. 11, p. 2048, 2020.

[6] P. Wen, Y. Gao, B. Chen et al., "Pan-cancer analysis of radiotherapy benefits and immune infiltration in multiple human cancers," Cancers, vol. 12, no. 4, p. 957, 2020.

[7] V. Izzi, M. N. Davis, and A. Naba, "Pan-cancer analysis of the genomic alterations and mutations of the matrisome," Cancers, vol. 12, no. 8, p. 2046, 2020.

[8] Y. Cui, W. Guo, Y. Li, J. Shi, S. Ma, and F. Guan, "Pan-cancer analysis identifies ESM1 as a novel oncogene for esophageal cancer," Esophagus : official journal of the Japan Esophageal Society, vol. 18, no. 2, pp. 326-338, 2021.

[9] L. Zhu, W. Wu, S. Jiang et al., "Pan-cancer analysis of the mitophagy-related protein PINK1 as a biomarker for the immunological and prognostic role," Frontiers in Oncology, vol. 10, p. 569887, 2020.

[10] Q. Ge, G. Li, J. Chen et al., "Immunological role and prognostic value of APBB1IP in pan-cancer analysis," Journal of Cancer, vol. 12, no. 2, pp. 595-610, 2021.

[11] L. Zhang, M. F. Walsh, S. Jairam et al., "Fumarate hydrataseFHc.1431_1433dupAAA (p.Lys477dup) variant is not associated with cancer including renal cell carcinoma," Human Mutation, vol. 41, no. 1, pp. 103-109, 2020.

[12] A. Ooi, "Advances in hereditary leiomyomatosis and renal cell carcinoma (HLRCC) research," Seminars in Cancer Biology, vol. 61, pp. 158-166, 2020.

[13] H. E. Yu, F. Wang, F. Yu et al., "Suppression of fumarate hydratase activity increases the efficacy of cisplatin-mediated chemotherapy in gastric cancer," Cell Death \& Disease, vol. 10, no. 6, p. 413, 2019.

[14] M. Sciacovelli, E. Gonçalves, T. I. Johnson et al., "Fumarate is an epigenetic modifier that elicits epithelial-to-mesenchymal transition," Nature, vol. 537, no. 7621, pp. 544-547, 2016.

[15] M. Suarez-Carmona, J. Lesage, D. Cataldo, and C. Gilles, "EMT and inflammation: inseparable actors of cancer progression," Molecular Oncology, vol. 11, no. 7, pp. 805-823, 2017.

[16] D. C. Hinshaw and L. A. Shevde, "The tumor microenvironment innately modulates cancer progression," Cancer Research, vol. 79, no. 18, pp. 4557-4566, 2019.

[17] X. Lei, Y. Lei, J. K. Li et al., "Immune cells within the tumor microenvironment: biological functions and roles in cancer immunotherapy," Cancer Letters, vol. 470, pp. 126-133, 2020.

[18] L. Yang and Y. Zhang, "Tumor-associated macrophages: from basic research to clinical application," Journal of Hematology \& Oncology, vol. 10, no. 1, p. 58, 2017.

[19] L. Cassetta and J. W. Pollard, "Tumor-associated macrophages," Current Biology : CB, vol. 30, no. 6, pp. R246-r248, 2020.

[20] Q. Ju, Y. J. Zhao, S. Ma et al., "Genome-wide analysis of prognostic-related lncRNAs, miRNAs and mRNAs forming a competing endogenous RNA network in lung squamous cell carcinoma," Journal of Cancer Research and Clinical Oncology, vol. 146, no. 7, pp. 1711-1723, 2020.

[21] Y. Zhao, H. Zhang, Q. Ju, X. Li, and Y. Zheng, "Comprehensive analysis of survival-related lncRNAs, miRNAs, and mRNAs forming a competing endogenous RNA network in gastric cancer," Frontiers in Genetics, vol. 12, p. 610501, 2021.

[22] S. A. Martin, M. Hewish, C. J. Lord, and A. Ashworth, "Genomic instability and the selection of treatments for cancer," The Journal of Pathology, vol. 220, pp. 281-289, 2010.

[23] R. Ijsselsteijn, J. G. Jansen, and N. de Wind, "DNA mismatch repair-dependent DNA damage responses and cancer," DNA Repair, vol. 93, p. 102923, 2020.

[24] J. Peña-Diaz and L. J. Rasmussen, "Approaches to diagnose DNA mismatch repair gene defects in cancer," DNA Repair, vol. 38, pp. 147-154, 2016.

[25] Y. Qin, X. Zheng, W. Gao, B. Wang, and Y. Wu, "Tumor microenvironment and immune-related therapies of head and neck squamous cell carcinoma," Molecular therapy oncolytics, vol. 20, pp. 342-351, 2021.

[26] Q. Ju, X. Li, H. Zhang, S. Yan, Y. Li, and Y. Zhao, "NFE2L2 is a potential prognostic biomarker and is correlated with immune infiltration in brain lower grade glioma: a pan-cancer analysis," Oxidative Medicine and Cellular Longevity, vol. 2020, Article ID 3580719, 26 pages, 2020. 
[27] Q. Ju, X. M. Li, H. Zhang, and Y. J. Zhao, "BRCA1-associated protein is a potential prognostic biomarker and is correlated with immune infiltration in liver hepatocellular carcinoma: a pan-cancer analysis," Frontiers in Molecular Biosciences, vol. 7, p. 573619, 2020.

[28] C. Schmidt, M. Sciacovelli, and C. Frezza, "Fumarate hydratase in cancer: a multifaceted tumour suppressor," Seminars in Cell \& Developmental Biology, vol. 98, pp. 15-25, 2020.

[29] Z. Cai, C. F. Li, F. Han et al., "Phosphorylation of PDHA by AMPK drives TCA cycle to promote cancer metastasis," Molecular Cell, vol. 80, no. 2, pp. 263-278.e7, 2020.

[30] N. M. Anderson, P. Mucka, J. G. Kern, and H. Feng, “The emerging role and targetability of the TCA cycle in cancer metabolism," Protein \& Cell, vol. 9, no. 2, pp. 216-237, 2018.

[31] S. Zhou, Y. Yan, X. Chen et al., "Roles of highly expressed PAICS in lung adenocarcinoma," Gene, vol. 692, pp. 1-8, 2019.

[32] Y. Yan, Z. Xu, X. Hu et al., "SNCA is a functionally lowexpressed gene in lung adenocarcinoma," Genes, vol. 9, no. 1, p. 16, 2018.

[33] U. Ben-David, R. Beroukhim, and T. R. Golub, "Genomic evolution of cancer models: perils and opportunities," Nature Reviews. Cancer, vol. 19, no. 2, pp. 97-109, 2019.

[34] S. Negrini, V. G. Gorgoulis, and T. D. Halazonetis, "Genomic instability - an evolving hallmark of cancer," Nature Reviews. Molecular Cell Biology, vol. 11, no. 3, pp. 220-228, 2010.

[35] D. L. Keefe, "Telomeres and genomic instability during early development," European Journal of Medical Genetics, vol. 63, no. 2, p. 103638, 2020.

[36] E. Sanz-Garcia, G. Argiles, E. Elez, and J. Tabernero, "BRAF mutant colorectal cancer: prognosis, treatment, and new perspectives," Annals of Oncology : official journal of the European Society for Medical Oncology, vol. 28, no. 11, pp. 2648-2657, 2017.

[37] M. Baretti and D. T. Le, "DNA mismatch repair in cancer," Pharmacology \& Therapeutics, vol. 189, pp. 45-62, 2018.

[38] P. Kancherla, M. Daneshvar, R. A. Sager, M. Mollapour, and G. Bratslavsky, "Fumarate hydratase as a therapeutic target in renal cancer," Expert Opinion on Therapeutic Targets, vol. 24, no. 9, pp. 923-936, 2020.

[39] K. E. Yost, A. T. Satpathy, D. K. Wells et al., "Clonal replacement of tumor-specific T cells following PD-1 blockade," Nature Medicine, vol. 25, no. 8, pp. 1251-1259, 2019.

[40] R. D. Schreiber, L. J. Old, and M. J. Smyth, "Cancer immunoediting: integrating immunity's roles in cancer suppression and promotion," Science, vol. 331, no. 6024, pp. 1565-1570, 2011.

[41] S. S. Wang, W. Liu, D. Ly, H. Xu, L. Qu, and L. Zhang, "Tumor-infiltrating B cells: their role and application in antitumor immunity in lung cancer," Cellular \& Molecular Immunology, vol. 16, no. 1, pp. 6-18, 2019.

[42] M. Horikawa, V. Minard-Colin, T. Matsushita, and T. F. Tedder, "Regulatory B cell production of IL-10 inhibits lymphoma depletion during CD20 immunotherapy in mice," The Journal of Clinical Investigation, vol. 121, no. 11, pp. 4268-4280, 2011.

[43] L. Zhang, J. R. Conejo-Garcia, D. Katsaros et al., "Intratumoral T cells, recurrence, and survival in epithelial ovarian cancer," The New England Journal of Medicine, vol. 348, no. 3, pp. 203-213, 2003.

[44] E. B. Eruslanov, P. S. Bhojnagarwala, J. G. Quatromoni et al., "Tumor-associated neutrophils stimulate $\mathrm{T}$ cell responses in early-stage human lung cancer," The Journal of Clinical Investigation, vol. 124, no. 12, pp. 5466-5480, 2014.
[45] Y. Komohara, Y. Fujiwara, K. Ohnishi, and M. Takeya, "Tumor-associated macrophages: potential therapeutic targets for anti-cancer therapy," Advanced Drug Delivery Reviews, vol. 99, pp. 180-185, 2016.

[46] F. Veglia and D. I. Gabrilovich, "Dendritic cells in cancer: the role revisited," Current Opinion in Immunology, vol. 45, pp. 43-51, 2017.

[47] M. Hansen and M. H. Andersen, "The role of dendritic cells in cancer," Seminars in Immunopathology, vol. 39, no. 3, pp. 307316, 2017.

[48] E. S. Knudsen, V. Kumarasamy, S. Chung et al., "Targeting dual signalling pathways in concert with immune checkpoints for the treatment of pancreatic cancer," Gut, vol. 70, no. 1, pp. 127-138, 2021.

[49] L. Long, C. Zhao, M. Ozarina, X. Zhao, J. Yang, and H. Chen, "Targeting immune checkpoints in lung cancer: current landscape and future prospects," Clinical Drug Investigation, vol. 39, no. 4, pp. 341-353, 2019. 